\title{
Integrable quadratic Hamiltonians on the Euclidean group of motions
}

\author{
James Biggs and William Holderbaum ${ }^{1}$
}

February 1, 2010

${ }^{1}$ J. Biggs is with the Department of Mechanical Engineering, University of Strathclyde, Glasgow,UK james.biggsestrath.ac.uk; W. Holderbaum is with School of Systems Engineering, University of Reading, Reading, UK w. holderbaumerdg.ac.uk 


\begin{abstract}
This paper tackles the problem of globally computing sub-Riemannian curves on the Euclidean group of motions $S E(3)$. In particular we derive a global result for special sub-Riemannian curves for which their Hamiltonian satisfies a particular condition. The sub-Riemannian curves in this paper are defined in the context of a constrained optimal control problem. The Maximum Principle is then applied to this problem to yield the appropriate left-invariant quadratic Hamiltonian. A number of integrable quadratic Hamiltonians are identified. We then proceed to derive convenient expressions for sub-Riemannian curves in $S E(3)$ that correspond to particular extremal curves. These equations are then used to compute sub-Riemannian curves that could potentially be used for motion planning of underwater vehicles.
\end{abstract}

Subject classification codes: 70Q05, 53C17.

Keywords: sub-Riemannian curves, Euclidean group of motions, Hamiltonian systems, Motion Planning. 


\subsection{Introduction}

This paper is motivated by the motion planning problem for rigid body systems with nonholonomic constraints $[1,2,3,4,5,6,7,8]$. The problem is formulated as a constrained optimal control problem on the Euclidean group of motions $S E(3)$ and is analogous to the problem of globally computing sub-Riemannian curves on this Lie group. In this context the horizontal constraint of the sub-Riemannian curve on $S E(3)$ is analogous to the velocity constraint on the rigid body. Furthermore, these sub-Riemannian curves are analogous to rigid body motions that are locally optimal with respect to minimizing a quadratic cost function of linear and/or angular velocity components. Moreover, the resulting rigid body motions are optimal for small terminal time $T$ [9] pg. 216-219.

These sub-Riemannian problems have generally been tackled using a variational (Lagrangian) approach, see [5, 10, 11, 12, 6, 13, 2], however, in this paper the problem is defined in the context of optimal control. An application of the Maximum Principle to this problem then yields a Hamiltonian rather than a EulerLagrange formulation. This formulation is advantageous as the sub-Riemannian problem can be treated globally through the coordinate free Maximum Principle of optimal control $[9,15]$. Apart from the original example of Brockett on the Heisenberg group [11] and the particular sub-Riemannian curves (called $\mathfrak{p}$ curves) defined on 3-dimensional Lie groups in [14], most of the studies of subRiemannian curves are local. Furthermore, this paper considers special cases of this problem where the optimal control problem lifts to integrable Hamiltonian systems.

The integrable Hamiltonian systems considered in this paper are important special cases of this problem as the extremals can be explicitly solved in exact form. Furthermore, since the behavior of an integrable Hamiltonian system is always periodic or quasi-periodic, an integrable system cannot display chaotic behavior [16] pg. 289. This suggests that motion planning problems that lift to integrable Hamiltonian systems will compose of regular motions. Previous work has identified integrable cases of Kirchhoff's equations [17] (topologically equivalent to the Hamiltonian lift of the Riemannian problem on $S E(3)$ ) and Hamiltonians corresponding to elastic curves on $S E(3)$ in $[20,21,9,18]$. In this paper several integrable quadratic Hamiltonians corresponding to sub-Riemannian curves on $S E(3)$ are identified. One particular integrable Hamiltonian considered corresponds to the motion planning problem for the underwater vehicle posed in [19]. Due to the practical relevance of this Hamiltonian we solve this particular case in detail. 
Following this we derive convenient expressions relating extremal curves that satisfy a particular condition to their corresponding curves $g(t) \in S E(3)$. This integration follows a similar procedure to that described for left-invariant Hamiltonian systems on the orthogonal group $S O(3)$ in [9] pg. 436-441, however, we further exploit an additional constant of motion to obtain convenient expressions for the projection of extremal curves (that satisfy a certain condition ) onto $\mathbb{R}^{3}$. Finally, using these equations we compute a set of sub-Riemannian curves corresponding to a particular non-trivial solution of an integrable quadratic Hamiltonian. These particular sub-Riemannian curves corresponds to helical motions that could be useful for planning ascending and descending motions for underwater vehicles [19].

\section{2 sub-Riemannian curves on $S E(3)$}

In this section the sub-Riemannian curves $g(t) \in S E(3)$ are defined in the context of an optimal control problem. We note that the element $g(t) \in S E(3)$ can be decomposed into $\hat{\gamma}=[x, y, z]^{T} \in \mathbb{R}^{3}$ and $R \in S O(3)$ such that $g(t)=\left(\begin{array}{ll}1 & 0 \\ \hat{\gamma} & R\end{array}\right)$. We define the basis of the Lie algebra $\mathfrak{s e}(3)$ by the elements $B_{1}, B_{2}, B_{3}, A_{1}, A_{2}, A_{3} \in$ $\mathfrak{s e}(3)$. The Lie algebra $\mathfrak{s e}(3)$ admits a direct sum decomposition $\mathfrak{s e}(3)=\mathfrak{p} \oplus \mathfrak{k}$ such that $B_{1}, B_{2}, B_{3} \in \mathfrak{p}$ and $A_{1}, A_{2}, A_{3} \in \mathfrak{k}$ and where the Lie bracket $[\cdot, \cdot]=X Y-Y X$ satisfies the following commutative table:

\begin{tabular}{|c|c|c|c|c|c|c|}
\hline$[]$, & $A_{1}$ & $A_{2}$ & $A_{3}$ & $B_{1}$ & $B_{2}$ & $B_{3}$ \\
\hline$A_{1}$ & 0 & $A_{3}$ & $-A_{2}$ & 0 & $B_{3}$ & $-B_{2}$ \\
$A_{2}$ & $-A_{3}$ & 0 & $A_{1}$ & $-B_{3}$ & 0 & $B_{1}$ \\
$A_{3}$ & $A_{2}$ & $-A_{1}$ & 0 & $B_{2}$ & $-B_{1}$ & 0 \\
$B_{1}$ & 0 & $B_{3}$ & $-B_{2}$ & 0 & 0 & 0 \\
$B_{2}$ & $-B_{3}$ & 0 & $B_{1}$ & 0 & 0 & 0 \\
$B_{3}$ & $B_{2}$ & $-B_{1}$ & 0 & 0 & 0 & 0 \\
\hline
\end{tabular}

A sub-Riemannian curve is then one that minimizes the energy with respect to the fiber inner product or that minimizes the length of the curve (the two yield the same curves through the Cauchy-Scwartz inequality, see [7] pg. 6-7). However, it is more convenient to minimize the energy and define a sub-Riemannian curve in the context of an optimal control problem on $S E(3)$ (see also [7], [5], [6], [13]): 
Definition 1 A sub-Riemannian curve $g(t) \in S E(3)$ with the left-invariant horizontal distribution, $D$ :

$$
\frac{d g(t)}{d t}=g(t)\left(\sum_{i=1}^{s} v_{i} B_{i}+\sum_{i=1}^{r} u_{i} A_{i}\right)
$$

minimizes the energy of a horizontal curve:

$$
J=\frac{1}{2} \int_{0}^{T} \sum_{i=1}^{s} m_{i} v_{i}^{2}+\sum_{i=1}^{r} c_{i} u_{i}^{2} d t
$$

subject to the given boundary conditions $g(0)=g_{0}$ and $g(T)=g_{T}$ and where $c_{i}, m_{i}$ are constants and $v_{i}, u_{i}$ are measurable and bounded functions.

Computing sub-Riemannian curves can then be naturally viewed as an optimal control problem with $u(t)=\left(v_{1}, \ldots, v_{s}, u_{1}, \ldots, u_{r}\right)$ playing the role of control functions where $s \leqslant 3$ and $r \leqslant 3$ are the number of controls in the translational and rotational directions respectively. If $s+r$ is equal to 6 , the constraint (1) is holonomic, which means that every path in $S E(3)$ is equivalently a trajectory of (1). If $s+r$ is less than 6, equation (1) describes a horizontal constraint and $g(t) \in S E(3)$ are sub-Riemannian curves. If we associate (1) with a kinematic control system where $v_{i}, u_{i}$ are the velocity controls and if $s+r<6$ then (1) is equivalently a nonholonomic constraint.

\subsection{Hamiltonian lift on SE(3)}

The application of the coordinate free Maximum Principle to optimal control problems of this type are well known, see [15], [9]. In this section we give a brief overview of this and show that the Hamiltonian corresponding to a subRiemannian curve on $S E(3)$ is quadratic. As the Hamiltonian is left-invariant the cotangent bundle $T^{*} S E(3)$ can be realized as the direct product $S E(3) \times \mathfrak{s e}(3)^{*}$ where $\mathfrak{s e}(3)^{*}$ is the dual of the Lie algebra $\mathfrak{s e}(3)$ of $S E(3)$. Therefore, the original Hamiltonian defined on $T^{*} S E(3)$ can be expressed as a reduced Hamiltonian on the dual of the Lie algebra $\mathfrak{s e}(3)^{*}$ as $T^{*} S E(3) / S E(3) \cong \mathfrak{s e}(3)^{*}$. The appropriate Hamiltonian for the horizontal curve (1) with respect to minimizing the horizontal curves energy (2) is given by (see [5] pg. 329-358 for details):

$$
\left.H(p, u, g)=\sum_{i=1}^{s} v_{i} p\left(g(t) B_{i}\right)+\sum_{i=1}^{r} u_{i} p\left(g(t) A_{i}\right)-\rho_{0} \frac{1}{2}\left(\sum_{i=1}^{s} m_{i} v_{i}^{2}+\sum_{i=1}^{r} c_{i} u_{i}^{2}\right)\right)
$$


where $p \in T^{*} S E(3)$ and $\rho_{0}=1$ for regular extremals and $\rho_{0}=0$ for abnormal extremals. In this paper we consider only the regular extremals, therefore we set $\rho_{0}=1$. The Hamiltonian (3) defined on $T^{*} S E(3)$ can be expressed as a reduced Hamiltonian on the dual of the Lie algebra $\mathfrak{s e}(3)^{*}$. It follows that $p\left(g(t) A_{i}\right)=$ $\hat{p}\left(A_{i}\right)$ for any $p=(g(t), \hat{p})$ and any $A_{i} \in \mathfrak{s e}(3), p\left(g(t) B_{i}\right)=\hat{p}\left(B_{i}\right)$ for any $p=$ $(g(t), \hat{p})$ and any $B_{i} \in \mathfrak{s e}(3)$. Defining the extremal (linear) functions explicitly as $M_{i}=\hat{p}\left(A_{i}\right), p_{i}=\hat{p}\left(B_{i}\right)$, where $\hat{p} \in \mathfrak{s e}^{*}(3)$ the Hamiltonian (3) can be expressed on $\mathfrak{s e}(3)^{*}$ as

$$
\left.H=\sum_{i=1}^{s} v_{i} p_{i}+\sum_{i=1}^{r} u_{i} M_{i}-\frac{1}{2}\left(\sum_{i=1}^{s} m_{i} v_{i}^{2}+\sum_{i=1}^{r} c_{i} u_{i}^{2}\right)\right)
$$

Through the Maximum Principle and the fact that the control Hamiltonian (4) is a concave function of the control functions $u_{i}, v_{i}$, it follows by calculating $\frac{\partial H}{\partial u_{i}}=0$ and $\frac{\partial H}{\partial v_{i}}=0$ that the optimal controls are:

$$
u_{i}^{*}=\frac{1}{c_{i}} M_{i}, \quad v_{i}^{*}=\frac{1}{m_{i}} p_{i}
$$

where $i=1,2,3$. Substituting (5) back into (4) gives the appropriate left-invariant quadratic Hamiltonian:

$$
H=\frac{1}{2}\left(\sum_{i=1}^{s} \frac{p_{i}^{2}}{m_{i}}+\sum_{i=1}^{r} \frac{M_{i}^{2}}{c_{i}}\right)
$$

where $M_{i}$ and $p_{i}$ are the extremal curves. For each quadratic Hamiltonian (6), the corresponding vector fields are calculated using the Poisson bracket $\{\hat{p}(\cdot), \hat{p}(\cdot)\}=$ $-\hat{p}([\cdot, \cdot])$ where $(\cdot) \in \mathfrak{s e}(3)$. Then the Hamiltonian vector fields are given by:

$$
\frac{d(\cdot)}{d t}=\{\cdot, H\}
$$

where $(\cdot) \in \mathfrak{s e}(3)^{*}$. Finally, substituting (5) into (1) yields:

$$
\frac{d g(t)}{d t}=g(t) \nabla H
$$

where $\nabla H$ is the gradient of the Hamiltonian and $g(t) \in S E(3)$ are the sub-Riemannian curves. 


\subsection{Integrable quadratic Hamiltonians on $S E(3)$}

To explicitly solve the sub-Riemannian curves $g(t) \in S E(3)$ it is first necessary to solve equation (7). Before proceeding to try and explicitly solve (7) it is sensible to ask if analytic solutions exist, that is, are the Hamiltonian vector fields integrable. Specifically, the integrability of left-invariant Hamiltonian systems defined on $S E(3)$ has been detailed in [9] pg. 407-453 and we summarize the essential results in the following:

For convenience of exposition we define the basis for $\mathfrak{s o}(3)$ :

$$
E_{1}=\left(\begin{array}{ccc}
0 & 0 & 0 \\
0 & 0 & -1 \\
0 & 1 & 0
\end{array}\right), E_{2}=\left(\begin{array}{ccc}
0 & 0 & 1 \\
0 & 0 & 0 \\
-1 & 0 & 0
\end{array}\right), E_{3}=\left(\begin{array}{ccc}
0 & -1 & 0 \\
1 & 0 & 0 \\
0 & 0 & 0
\end{array}\right)
$$

then for left-invariant Hamiltonians on $S E(3)$ we state the following facts:

Theorem 1 The quantities $\pi \in \mathfrak{s o}(3)$ and $\rho \in \mathfrak{s o}(3)$ defined by the equations:

$$
R P R^{-1}=\rho
$$

and

$$
R M R^{-1}+\left[\gamma, R P R^{-1}\right]=\pi
$$

are conserved quantities for any left-invariant Hamiltonian systems on SE(3) where

$$
\begin{gathered}
M=M_{1} E_{1}+M_{2} E_{2}+M_{3} E_{3} \\
P=p_{1} E_{1}+p_{2} E_{2}+p_{3} E_{3} \\
\gamma=x E_{1}+y E_{2}+z E_{3}
\end{gathered}
$$

Proof. see [9] pg. 391-392. The conditions (10) and (11) imply the existence of two constant functions on $\mathfrak{s e}^{*}(3)$ :

$$
p_{1}^{2}+p_{2}^{2}+p_{3}^{2}=-\frac{1}{2} \operatorname{trace}\left(\rho^{2}\right)
$$

and

$$
M_{1} p_{1}+M_{2} p_{2}+M_{3} p_{3}=-\frac{1}{2} \operatorname{trace}(\rho \pi)
$$

for convenience we denote the constant $I_{2}=-\frac{1}{2} \operatorname{trace}\left(\rho^{2}\right)$ and $I_{3}=-\frac{1}{2} \operatorname{trace}(\rho \pi)$. $I_{2}$ and $I_{3}$ are Casimir functions and are in involution with any other constant function on $\mathfrak{s e}^{*}(3)$ i.e. $\left\{I_{2}, H\right\}=0,\left\{I_{3}, H\right\}=0$ and $\left\{I_{2}, I_{3}\right\}=0$. For left-invariant 
Hamiltonian systems defined on $S E(3)$ we can then be more specific about integrability, following the arguments posed in [9] pg. 458-459 we state the following Lemma:

Lemma 1 For any left (respectively right) invariant Hamiltonian system defined on $S E(3)$, there exist five functionally independent constants of motion $\varphi_{1}=$ $H, \varphi_{2}=I_{2}, \varphi_{3}=I_{3}, \varphi_{4}, \varphi_{5}$, where the constants of motion $\varphi_{4}, \varphi_{5}$ correspond to right-invariant vector fields.

As the Hamiltonian system is defined on the 12-D cotangent bundle $T^{*} S E(3) \cong$ $S E(3) \times \mathfrak{s e}^{*}(3)$ six constants of motion are required for integrability [9] pg. 444446. Therefore, we require an additional constant of motion for the system to be integrable. Firstly, we note that in the case that the curves are unconstrained $(s+$ $r=6$ ) the corresponding Hamiltonian vector fields are topologically equivalent to the Kirchhoff equations. The integrable cases of these equations have been studied in [17]. In this paper we identify a number of integrable quadratic Hamiltonians on $S E(3)$ which correspond to sub-Riemannian curves.

\subsubsection{Example cases of integrable quadratic Hamiltonians}

In this subsection we identify explicitly a number of integrable quadratic Hamiltonians on $S E(3)$ that correspond to certain sub-Riemannian curves.

\section{Integrable Hamiltonian 1:}

To begin with we look at a Hamiltonian that corresponds to a particular type of sub-Riemannian curve (coined $\mathfrak{p}$-curves in [14]). These particular sub-Riemannian problems are concerned with a left-invariant metric defined on $\mathfrak{p}$. Moreover, given a positive definite quadratic form $\langle\cdot, \cdot\rangle$ defined on $\mathfrak{p}$ we consider the problem of minimizing the integral

$$
\frac{1}{2} \int_{0}^{T}\left\langle g(t)^{-1} \frac{d g(t)}{d t}, g(t)^{-1} \frac{d g(t)}{d t}\right\rangle d t
$$

among all continuous curves in $S E(3)$ defined on an interval $[0, T]$ that satisfies $g(t)^{-1} \frac{d g(t)}{d t}=p(t)$ with $p(t) \in \mathfrak{p}$ and which satisfy the fixed boundary conditions $g(0)=g_{0}$ and $g(T)=g_{T}$. A Hamiltonian corresponding to a $\mathfrak{p}$-curve is:

$$
H=\frac{1}{2}\left(p_{1}^{2}+p_{2}^{2}+p_{3}^{2}\right)
$$


and the corresponding Hamiltonian vector fields are $\dot{p}_{1}=\dot{p}_{2}=\dot{p}_{3}=\dot{M}_{1}=\dot{M}_{2}=$ $\dot{M}_{3}=0$ and therefore integrable. It is then easily shown that the extremal curves are constant and the sub-Riemannian curves are straight lines in $\mathbb{R}^{3}$.

\section{Integrable Hamiltonian 2:}

We consider a Hamiltonian that corresponds to the case where $g(t)^{-1} \frac{d g(t)}{d t} \in k(t)$ with $k(t) \in \mathfrak{k}$. The reachable set of this case is equal to the space of all rotations of $\mathbb{R}^{3}$ and corresponds to the Hamiltonian:

$$
H=\frac{1}{2}\left(\frac{M_{1}^{2}}{c_{1}}+\frac{M_{2}^{2}}{c_{2}}+\frac{M_{3}^{2}}{c_{3}}\right)
$$

The Hamiltonian vector fields corresponding to the Hamiltonian (17) are topologically equivalent to the Euler equations [5]. In addition substituting $p_{i}=0$ into (11) yields $R M R^{-1}=$ constant. It follows that

$$
-\frac{1}{2} \operatorname{trace}\left(R M R^{-1}\right)=M_{1}^{2}+M_{2}^{2}+M_{3}^{2}
$$

is a constant and therefore with (17) there are two constants of motion. Therefore, the Hamiltonian system is integrable and the solutions are elliptic functions of time [22]: they are single-valued on the plane of complex time and their singular points are poles.

\section{Integrable Hamiltonian 3:}

In this case we consider a Hamiltonian that corresponds to a sub-Riemannian problem that is controllable on $S E(3)$, that is, any two points $g_{0}$ and $g_{T}$ in $S E$ (3) can be connected by a sub-Riemannian curve. We consider a sub-Riemannian curve $g(t) \in S E(3)$ with the horizontal distribution $D$ defined by:

$$
\frac{d g(t)}{d t}=g(t)\left(\sum_{i=1}^{3} v_{i} B_{i}+u_{1} A_{1}\right)
$$

that minimizes the energy of the horizontal curve:

$$
J=\frac{1}{2} \int_{0}^{T} \sum_{i=1}^{3} m_{i} v_{i}^{2}+c_{1} u_{1}^{2} d t
$$

subject to the given boundary conditions $g(0)=g_{0}$ and $g(T)=g_{T}$ and where $c_{i}, m_{i}$ are constants and $u(t)=\left(v_{1}, v_{2}, v_{3}, u_{1}\right)$ are measurable and bounded functions. 
The corresponding quadratic Hamiltonian is then:

$$
H=\frac{1}{2}\left(\frac{M_{1}^{2}}{c_{1}}+\frac{p_{1}^{2}}{m_{1}}+\frac{p_{2}^{2}}{m_{2}}+\frac{p_{3}^{2}}{m_{3}}\right)
$$

It is easy to show that $\frac{d p_{1}}{d t}=0$ and therefore the system is integrable.

\section{Integrable Hamiltonian 4}

Finally, we identify a case where the Hamiltonian vector fields are integrable under a specific symmetry assumption. This sub-Riemannian problem is completely analogous to the optimal kinematic control problem for an underwater vehicle posed in [19]. Due to the practical relevance of this case it is analyzed in detail. This sub-Riemannian problem has horizontal distribution:

$$
\frac{d g(t)}{d t}=g(t)\left(v_{1} B_{1}+\sum_{i=1}^{3} u_{i} A_{i}\right)
$$

that minimizes the energy of the horizontal curve:

$$
J=\frac{1}{2} \int_{0}^{T} m_{1} v_{1}^{2}+\sum_{i=1}^{3} c_{i} u_{i}^{2} d t
$$

subject to the given boundary conditions $g(0)=g_{0}$ and $g(T)=g_{T}$ and where $c_{1}, c_{2}, c_{3}, m_{1}$ are constants and $u(t)=\left(v_{1}, u_{1}, u_{2}, u_{3}\right)$ are measurable and bounded functions. The constraint (22) is analogous to a conventional underwater vehicle whose lateral motions are quickly damped out due to damping. The appropriate quadratic Hamiltonian on $S E(3)$ is then:

$$
H=\frac{1}{2}\left(\frac{p_{1}^{2}}{m_{1}}+\frac{M_{1}^{2}}{c_{1}}+\frac{M_{2}^{2}}{c_{2}}+\frac{M_{3}^{2}}{c_{3}}\right)
$$


with the corresponding Hamiltonian vector fields equal to:

$$
\left\{\begin{array}{l}
\frac{d M_{1}}{d t}=\frac{-M_{2} M_{3}}{c_{2}}+\frac{M_{2} M_{3}}{c_{3}} \\
\frac{d M_{2}}{d t}=\frac{M_{1} M_{3}}{c_{1}}-\frac{M_{1} M_{3}}{c_{3}}+\frac{p_{1} p_{3}}{m_{1}} \\
\frac{d M_{3}}{d t}=\frac{-M_{1} M_{2}}{c_{1}}+\frac{M_{1} M_{2}}{c_{2}}-\frac{p_{1} p_{2}}{m_{1}} \\
\frac{d p_{1}}{d t}=\frac{-M_{2} p_{3}}{c_{2}}+\frac{p_{2} M_{3}}{c_{3}} \\
\frac{d p_{2}}{d t}=\frac{M_{1} p_{3}}{c_{1}}-\frac{p_{1} M_{3}}{c_{3}} \\
\frac{d p_{3}}{d t}=-\frac{M_{1} p_{2}}{c_{1}}+\frac{p_{1} M_{2}}{c_{2}}
\end{array}\right.
$$

where $M_{1}, M_{2}, M_{3}, p_{1}, p_{2}, p_{3} \in \mathfrak{s e}^{*}(3)$ are the extremal curves. We proceed by using a symmetry argument analogous to the symmetric top [9], setting $c_{2}=c_{3}$ gives $\frac{d M_{1}}{d t}=0$ thus yielding the extra integral of motion required for integrability.

\subsection{Projecting particular extremals onto $S E(3)$}

This section derives convenient expressions relating extremal curves that satisfy the condition $p_{2}^{2}+p_{3}^{2}>0$ for all $t$ onto their corresponding locally optimal paths $g(t) \in S E(3)$. This derivation exploits the conserved quantities inherent in all left-invariant Hamiltonian systems on $S E(3)$. For convenience the Hamiltonian equations (8) are split into a translational part $\hat{\gamma} \in \mathbb{R}^{3}$ and a rotational part $R \in$ $S O(3)$ :

$$
\frac{d \hat{\gamma}}{d t}=R\left[\begin{array}{lll}
\frac{\partial H}{\partial p_{1}} & \frac{\partial H}{\partial p_{2}} & \frac{\partial H}{\partial p_{3}}
\end{array}\right]^{T}
$$

and

$$
\frac{d R}{d t}=R\left(\frac{\partial H}{\partial M_{1}} E_{1}+\frac{\partial H}{\partial M_{2}} E_{2}+\frac{\partial H}{\partial M_{3}} E_{3}\right)
$$

\subsubsection{Projecting particular extremals onto $S O(3)$}

Proceeding to solve the differential equation (27) for the rotational component $R \in S O(3)$ in terms of the extremal curves that satisfy a certain condition, we 
make use of the conservation laws stated in Theorem 1. This projection onto $S O(3)$ is similar to that described in [9] with some modifications that yield more compact expressions. For convenience define a constant $K^{2}=I_{2}$ where $I_{2}$ is the Casimir function (13) and assume $K \neq 0$. If this assumption does not hold then the equations of motion degenerate to pure rotations (zero translations), so the motions considered here are those which comprise of both rotations and translations. In terms of applications to the motion planning problem for autonomous underwater vehicles and unmanned air vehicles this assumption is acceptable as the vehicles will be propelled in a forward (translational) direction. Following the procedure outlined in [9] pg. 436-440 and for a given $P$ there always exists an element $R_{0} \in S O(3)$ such that:

$$
R_{0} P R_{0}^{-1}=K E_{1}
$$

This fact implies that any orbit $R P R^{-1}=\rho$ is conjugate to $\rho=K E_{1}$ and therefore it suffices to integrate the particular orbit:

$$
R P R^{-1}=K E_{1}
$$

Then let $\phi_{1}, \phi_{2}, \phi_{3}$ (Euler angles) denote the coordinates of a point in $S O(3)$ according to the formula:

$$
R=\exp \left(\phi_{1} E_{1}\right) \exp \left(\phi_{2} E_{2}\right) \exp \left(\phi_{3} E_{1}\right)
$$

with the appropriate ranges of the angles defined by $\phi_{1}, \phi_{3} \in(-\pi, \pi]$ and $\phi_{2} \in$ $[0, \pi]$. These Euler angles can be expressed completely in terms of elements in the dual of the Lie algebra provided that they satisfy a specific condition this is described in the following Theorem:

Theorem 2 The Euler angles describing the evolution of the rotation matrix $R \in$ $S O(3)$, can be completely expressed in terms of the extremal curves, such that:

$$
\begin{gathered}
\dot{\phi}_{1}=K\left(\frac{\left(p_{2} \frac{\partial H}{\partial M_{2}}\right)+\left(p_{3} \frac{\partial H}{\partial M_{3}}\right)}{p_{2}^{2}+p_{3}^{2}}\right) \\
\phi_{2}=\arccos \left(\frac{p_{1}}{K}\right) \\
\phi_{3}=\operatorname{atan} 2\left(p_{2}, p_{3}\right)
\end{gathered}
$$

where $H$ is any left-invariant Hamiltonian on $\mathfrak{s e}^{*}(3)$ provided the extremal curves satisfy the condition $p_{2}^{2}+p_{3}^{2}>0$ for all $t$. 
Proof. Using the equation (29) write:

$$
P=K R^{-1} E_{1} R
$$

substituting (30) into (32) yields:

$$
P=K\left(\begin{array}{ccc}
0 & -\cos \phi_{3} \sin \phi_{2} & \sin \phi_{2} \sin \phi_{3} \\
\cos \phi_{3} \sin \phi_{2} & 0 & -\cos \phi_{2} \\
-\sin \phi_{2} \sin \phi_{3} & \cos \phi_{2} & 0
\end{array}\right)
$$

then equating (33) to $P$ in (12) yields:

$$
p_{1}=K \cos \phi_{2}
$$

furthermore

$$
\begin{aligned}
& p_{2}=K \sin \phi_{2} \sin \phi_{3} \\
& p_{3}=K \sin \phi_{2} \cos \phi_{3}
\end{aligned}
$$

dividing $p_{2}$ by $p_{3}$ in (35) gives $\phi_{3}$ in terms of the extremal solutions:

$$
\frac{p_{2}}{p_{3}}=\tan \phi_{3}
$$

then for the Euler angle $\phi_{3} \in(-\pi, \pi]$ it follows that

$$
\sin \phi_{3}=\frac{p_{2}}{\sqrt{p_{2}^{2}+p_{3}^{2}}}, \cos \phi_{3}=\frac{p_{3}}{\sqrt{p_{2}^{2}+p_{3}^{2}}}
$$

To obtain an expression for $\phi_{1}$ we follow an analogous procedure to that used in [9] pg. 436-440. Finally, there are singularities in the equations (31) at $p_{2}^{2}+p_{3}^{2}=0$ and therefore these equations are valid provided $p_{2}^{2}+p_{3}^{2}>0$ for all $t$.

In addition for $\phi_{2} \in[0, \pi]$ then from (31) we can write:

$$
\sin \phi_{2}=\sqrt{1-\frac{p_{1}^{2}}{K^{2}}}=\frac{\sqrt{p_{2}^{2}+p_{3}^{2}}}{K}
$$

Then substituting (31), (38), (37) into (30) we state the following Lemma:

Lemma 2 The projection of particular extremal curves (that satisfy the condition $p_{2}^{2}+p_{3}^{2}>0$ for all $\left.t\right)$ onto $S O(3)$ are of the form:

$$
R=\left(\begin{array}{ccc}
b & a c & a d \\
a e & d f-b c e & -b d e-f c \\
-a f & d e+b c f & b d f-e c
\end{array}\right)
$$


where:

$$
\begin{aligned}
& a=\frac{\sqrt{p_{2}^{2}+p_{3}^{2}}}{K}, \quad b=\frac{p_{1}}{K}, \quad c=\frac{p_{2}}{\sqrt{p_{2}^{2}+p_{3}^{2}}}, \quad d=\frac{p_{3}}{\sqrt{p_{2}^{2}+p_{3}^{2}}} \\
& e=\sin \left(\int_{0}^{T} K\left(\frac{\left(p_{2} \frac{\partial H}{\partial M_{2}}\right)+\left(p_{3} \frac{\partial H}{\partial M_{3}}\right)}{p_{2}^{2}+p_{3}^{2}}\right) d t\right), \\
& f=\cos \left(\int_{0}^{T} K\left(\frac{\left(p_{2} \frac{\partial H}{\partial M_{2}}\right)+\left(p_{3} \frac{\partial H}{\partial M_{3}}\right)}{p_{2}^{2}+p_{3}^{2}}\right) d t\right)
\end{aligned}
$$

Note that the constant of integration $C$ of the integral in $e$ and $f$ will depend on the initial boundary condition $\phi_{1}(0)=\phi_{1_{0}}$. Furthermore, this integral is defined if the function $\dot{\phi}_{1}$ is continuous on the domain $t \in[0, T]$, explicitly the integral is defined provided that $p_{2}^{2}+p_{3}^{2} \neq 0$ or equivalently $K \neq \pm p_{1}$ on the domain $t \in[0, T]$. In addition for integrable Hamiltonian systems we can often solve the extremal curves analytically. In this case it is useful to state a condition for the integral in $e$ and $f$ (40) to be defined and that can be easily evaluated. To this end, note that at the singularities $K= \pm p_{1}$ and therefore from the constraint (13) $p_{1}$ is either a maximum or a minimum. It follows that if the extremal solution $p_{1}$ satisfies the condition $\left.\frac{d p_{1}}{d t}\right|_{K= \pm p_{1}} \neq 0$ then the integral in $e$ and $f$ is defined for all time.

\subsubsection{Projection of the extremal curves onto $\mathbb{R}^{3}$}

In this subsection, we exploit the conservation law (11) to yield convenient expressions relating the path of the sub-Riemannian curve in terms of the extremals:

Theorem 3 The path $\hat{\gamma}=[x, y, z]^{T} \in \mathbb{R}^{3}$ traced by the sub-Riemannian curve $g(t) \in$ $S E(3)$ can be expressed completely in terms of particular extremal curves (that satisfy the condition $p_{2}^{2}+p_{3}^{2}>0$ for all $t$ ) as:

$$
\begin{gathered}
\frac{d x}{d t}=\frac{1}{K}\left(p_{1} \frac{\partial H}{\partial p_{1}}+p_{2} \frac{\partial H}{\partial p_{2}}+p_{3} \frac{\partial H}{\partial p_{3}}\right) \\
y=\frac{1}{K}\left(\alpha \sin \phi_{1}-\beta \cos \phi_{1}-s_{3}\right) \\
z=-\frac{1}{K}\left(\alpha \cos \phi_{1}+\beta \sin \phi_{1}-s_{2}\right)
\end{gathered}
$$


where $s_{2}$ and $s_{3}$ are constants dependent on the initial position $g(0)$ and initial value $M(0)$ and

$$
\begin{aligned}
& \alpha=\left(\frac{M_{3} p_{2}-M_{2} p_{3}}{\sqrt{p_{2}^{2}+p_{3}^{2}}}\right) \\
& \beta=\left(\frac{p_{1}\left(M_{3} p_{3}+M_{2} p_{2}\right)-M_{1}\left(p_{2}^{2}+p_{3}^{2}\right)}{K \sqrt{p_{2}^{2}+p_{3}^{2}}}\right)
\end{aligned}
$$

Proof.

Recall that the integration for the rotation matrix is with respect to the particular solution (29) therefore the conservation law (11) can be expressed as:

$$
R M R^{-1}+\left[\gamma, K E_{1}\right]=\pi
$$

where $\pi \in \mathfrak{s o}(3)$ is a constant matrix which we define as:

$$
\pi=s_{1} E_{1}+s_{2} E_{2}+s_{3} E_{3}
$$

then substituting (44) and (30) into (43) gives

$$
\begin{aligned}
& y=\frac{1}{K} \sin \phi_{1}\left(M_{3} \sin \phi_{3}-M_{2} \cos \phi_{3}\right) \\
& -\frac{1}{K}\left(\cos \phi_{1}\left(\cos \phi_{2}\left(M_{3} \cos \phi_{3}+M_{2} \sin \phi_{3}\right)-M_{1} \sin \phi_{2}\right)+s_{3}\right) \\
& z=\frac{1}{K} \cos \phi_{1}\left(M_{2} \cos \phi_{3}-M_{3} \sin \phi_{3}\right) \\
& -\frac{1}{K}\left(\sin \phi_{1}\left(\cos \phi_{2}\left(M_{3} \cos \phi_{3}+M_{2} \sin \phi_{3}\right)-M_{1} \sin \phi_{2}\right)-s_{2}\right)
\end{aligned}
$$

and it follows that substituting (37), (34) and (38) into (45) and simplifying yields $y$ and $z$ in (41). Finally, to obtain an equation for $x$, substitute the coordinate representation of $R$ (30) into the state space equation (26) to yield:

$$
\frac{d x}{d t}=\cos \phi_{2} \frac{\partial H}{\partial p_{1}}+\sin \phi_{2} \sin \phi_{3} \frac{\partial H}{\partial p_{2}}+\cos \phi_{3} \sin \phi_{2} \frac{\partial H}{\partial p_{3}}
$$

then on substituting the expressions (31), (38) and (37) into (46) and simplifying yields the equation for $x$ in (41). 
The equations (39) and (41) describe a particular solution $g(t) \in S E(3)$ with respect to the initial condition imposed by the assumption (29), however, if we let the solution of equation (8) be denoted by $g_{g e n} \in S E(3)$ and any arbitrary initial condition by $g_{\text {int }} \in S E(3)$, then given the particular solution $g(t) \in S E(3)$ the solution for arbitrary initial conditions is given by:

$$
g_{g e n}=g_{\text {int }} g(0)^{-1} g(t)
$$

Finally, using the equations derived in this paper we compute explicitly a nontrivial sub-Riemannian curve on $S E(3)$.

\subsection{Explicit solutions to a sub-Riemannian problem on $S E(3)$}

The integrable quadratic Hamiltonian 4 in Section 0.4 is of practical relevance to the optimal control of an autonomous underwater vehicle posed in [19]. For this reason we use the proceeding analysis to solve for a sub-Riemannian curve explicitly. In this case a symmetry assumption is required for integrability and we set $c_{2}=c_{3}=1$ for simplicity of exposition. In this case $\dot{M}_{1}=0$ and therefore $M_{1}$ is a constant which will be denoted as $\sigma$ then from (24) we have:

$$
2 H-\frac{p_{1}^{2}}{m_{1}}-\frac{\sigma^{2}}{c_{1}}=M_{2}^{2}+M_{3}^{2}
$$

then from (25) it is easily shown that:

$$
\left(\frac{d p_{1}}{d t}\right)^{2}=p_{2}^{2} M_{3}^{2}+p_{3}^{2} M_{2}^{2}-2 p_{2} p_{3} M_{2} M_{3}
$$

Using (13) write:

$$
I_{2}-p_{1}^{2}=p_{2}^{2}+p_{3}^{2}
$$

multiplying (50) with (48) gives:

$$
\left(I_{2}-p_{1}^{2}\right)\left(2 H-\frac{p_{1}^{2}}{m_{1}}-\frac{\sigma^{2}}{c_{1}}\right)=p_{2}^{2} M_{2}^{2}+p_{2}^{2} M_{3}^{2}+p_{3}^{2} M_{2}^{2}+p_{3}^{2} M_{3}^{2}
$$

additionally we use the Casimir function (14) and rearrange to get:

$$
I_{3}-p_{1} \sigma=p_{2} M_{2}+p_{3} M_{3}
$$


and squaring (52) yields:

$$
\left(I_{3}-p_{1} \sigma\right)^{2}=p_{2}^{2} M_{2}^{2}+p_{3}^{2} M_{3}^{2}+2 p_{2} M_{2} p_{3} M_{3}
$$

finally substituting (53) and (51) into (49) and simplifying gives the following quartic function:

$$
\left(\dot{p}_{1}\right)^{2}=\frac{p_{1}^{4}}{m_{1}}+\left(\frac{\sigma^{2}}{c_{1}}-\sigma^{2}-\frac{I_{2}}{m_{1}}-2 H\right) p_{1}^{2}+\left(2 I_{3} \sigma\right) p_{1}+\left(2 H I_{2}-I_{3}^{2}-\frac{I_{2} \sigma^{2}}{c_{1}}\right)
$$

The function (54) is a quartic function of $p_{1}$ and can therefore be solved by an elliptic function, see [23], however, this is a complex symbolic expression and is omitted. In the remainder of this example we consider a particular solution that corresponds to the poles of the elliptic function defined by (54), that is extremals that satisfy $\dot{p}_{1}=0$. We denote the solutions that satisfy this condition as $p_{c}$. The remaining extremal curves will then be expressed in terms of this constant. To solve for the remaining extremal curves $M_{2}$ and $M_{3}$ the Hamiltonian function (48) is used. This suggests using polar coordinates for $M_{2}$ and $M_{3}$ :

$$
M_{2}=r \sin \theta, \quad M_{3}=r \cos \theta
$$

$r$ is given by substituting (55) into (48):

$$
r=\left(2 H-\frac{p_{c}^{2}}{m_{1}}-\frac{\sigma^{2}}{c_{1}}\right)^{1 / 2}
$$

and $\theta$ is given as follows:

$$
\begin{gathered}
\theta=\arctan \left(\frac{M_{2}}{M_{3}}\right) \\
\dot{\theta}=\frac{M_{3} \dot{M}_{2}-M_{2} \dot{M}_{3}}{M_{2}^{2}+M_{3}^{2}}
\end{gathered}
$$

substituting in the values for $\dot{M}_{2}, \dot{M}_{3}$ and the equations (48) and (52) yields the constant $\dot{\theta}$ :

$$
\dot{\theta}=\frac{\sigma}{c_{1}}-\sigma+\frac{p_{c}\left(I_{3}-p_{c} \sigma\right)}{m_{1}\left(2 H-\frac{\sigma^{2}}{c_{1}}-\frac{p_{c}^{2}}{m_{1}}\right)}
$$

we define the constant $\beta=\dot{\theta}$ and therefore $\theta$ is linear in $t$ and we write $\theta=\beta t$ (assuming for simplicity that the constant of integration is 0 ). Then substituting 
the solutions $p_{1}=p_{c}, M_{1}=\sigma, M_{2}=r \sin \beta t, M_{3}=r \cos \beta t$ and the derivatives $\dot{M}_{2}=r \beta \cos \beta t, \dot{M}_{2}=-r \beta \sin \beta t$ into (25) yields:

$$
\begin{aligned}
& p_{2}=s \sin \beta t \\
& p_{3}=s \cos \beta t
\end{aligned}
$$

where

$$
s=\frac{m_{1}}{p_{c}}\left(r \beta-\sigma r\left(\frac{1}{c_{1}}-1\right)\right)
$$

In summary these particular extremal solutions are defined as:

$M_{1}=\sigma, \quad M_{2}=r \sin \beta t, \quad M_{3}=r \cos \beta t, \quad p_{1}=p_{c}, \quad p_{2}=s \sin \beta t, \quad p_{3}=s \cos \beta t$,

where $p_{c}, \sigma, r, s, \beta$ are constants. The next step in the procedure is to project the extremals (62) onto $S E(3)$ to yield the corresponding sub-Riemannian curve. Assuming $s \neq 0$ the extremals (62) satisfy the condition of $p_{2}^{2}+p_{3}^{2}>0$ and Theorem 3 can be applied. Assuming for simplicity that the constants of integration are zero, the sub-Riemannian curves $g(t) \in S E(3)$ trace helical curves of the form:

$$
x=C_{1} t, \quad y=C_{2} \cos C_{3} t, \quad z=C_{2} \sin C_{3} t,
$$

where $C_{1}, C_{2}, C_{3}$ are constants defined by

$$
C_{1}=\frac{p_{c}^{2}}{K}, \quad C_{2}=-\frac{p_{c}\left(I_{3}-p_{c} \sigma\right)-\sigma s^{2}}{K^{2} s}, \quad C_{3}=K\left(\frac{I_{3}-p_{c} \sigma}{s^{2}}\right)
$$

Finally, applying Lemma 2 to the extremal curves (62) the orthonormal frame rotates along the curve according to the rotation matrix

$R=\left(\begin{array}{ccc}\frac{p_{c}}{K} & \frac{s}{K} \sin \beta t & \frac{s}{K} \cos \beta t \\ \frac{s}{K} \sin C_{3} t & \cos \beta t \cos C_{3} t-\frac{p_{c}}{K} \sin \beta t \sin C_{3} t & -\frac{p_{c}}{K} \cos \beta t \sin C_{3} t-\sin \beta t \cos C_{3} t \\ -\frac{s}{K} \cos C_{3} t & \cos \beta t \sin C_{3} t+\frac{p_{c}}{K} \sin \beta t \cos C_{3} t & \frac{p_{c}}{K} \cos \beta t \cos C_{3} t-\sin \beta t \sin C_{3} t\end{array}\right)$

Therefore, equations (63) and (65) define a sub-Riemannian curve on $S E(3)$.

\subsection{Conclusion}

This paper computes globally defined sub-Riemannian curves on the Euclidean group of motions $S E(3)$ whose extremal curves satisfy a particular condition. 
The problem is conveniently formulated as a constrained optimal control problem and an application of the Maximum Principle lifts the problem to its corresponding Hamiltonian vector fields. Following this a number of sub-Riemannian curves that yield integrable quadratic Hamiltonians were identified and one particular case solved analytically. Furthermore, an illustration of how to project particular extremal curves down onto $S E(3)$ is given and applied to the particular case. The derived sub-Riemannian curves yield an analytic solution which (analogously) solves a motion planning problem for the underwater vehicle defined in [19]. These analytic solutions could potentially be used to plan ascending and descending motions for underwater vehicles or landing motions for airplanes. Future work will investigate the use of sub-Riemannian curves as reference trajectories for real engineering rigid body systems with nonholonomic constraints. This will include an analysis of the forces required to track these reference trajectories. 


\section{Bibliography}

[1] Sastry, S., 'Nonlinear systems'. Analysis, Stability and Control, Interdiscipliary Applied Mathematics, Systems and Control,Springer, 1999.

[2] Hussein, I. I., Bloch, A. M., 'Constrained Optimal Trajectory on the Group of Rigid Body Motions'. Proceedings of IEEE CDC-ECC,Seville, 2005.

[3] Sastry, S., Zexiang, Li., 'Robot Motion Planning with nonholonomic constraints'. Proceedings of the 28th CDC, Florida, 1989.

[4] Zefran, M., Kumar, V., Croke, C., 'On the generation of smooth three dimensional rigid body motions'. IEEE Trans. Robotics and Automation, vol. 14, pp. 579-589, 1995.

[5] Bloch, A. M., 'Nonholonomic Mechanics and Control'. Springer-Verlag, New York,2003.

[6] Bloch, A., Crouch, P., Ratui, T., 'Sub-Riemannian optimal control problems'. Fields Institute Communications, vol. 3, pp. 35-48, 1994.

[7] Montgomery, R., 'A Tour of Subriemannian Geometries, Their Geodesics and Applications'. Mathematical Surveys and Monographs, vol. 91, American Mathematical Society, 2002.

[8] Crouch, P., Silva Leite, F., 'The Dynamic interpolation problem: On Riemannian manifolds, Lie groups and Symmetric spaces'. Journal of Dynamical and control systems, Vol.1, No. 2, p177-202, 1995.

[9] Jurdjevic, V., 'Geometric Control Theory'. Advanced Studies in Mathematics, Cambridge University Press, 52,1997. 
[10] Brockett, R., Dai, L., 'Non-holonomic kinematics and the role of elliptic functions in constructive controllability'. in Nonholonomic Motion Planning, (Z.X Li and J. Canny, ed.), Amsterdam: Kluwer, 1992.

[11] Brockett, R., 'Control theory and singular Riemannian geometry'. New directions in Applied Mathematics, (P.J. Hilton and G. S. Young, eds.), Springer, Berlin pp. 11-27, 1981.

[12] Baillieul, J., 'Geometric Methods for nonlinear optimal control problems'. J. Optim. Theory Appl., 25, pp. 519-548, 1978.

[13] Silva Leite, F., Camarinha, M., and Crouch, P., 'Elastic Curves as Solutions of Riemannian and Sub-Riemannian Control Problems'. Mathematics of Control, Signals, and Systems, 13: 140-155, 2000.

[14] Jurdjevic, V., 'Hamiltonian point of view of non-Euclidean geometry and elliptic functions'. Systems and Control Letters, 43, pp. 25-41, 2005.

[15] Sussmann, H. J., 'An introduction to the coordinate-free maximum principle'. In Geometry of Feedback and Optimal Control,B. Jakubczyk and W. Respondek Eds., Marcel Dekker, New York, pp. 463-557, 1997.

[16] Hilborn, R. C., 'Chaos and Nonlinear Dynamics: an introdution for scientists and engineers'. Oxford University Press, 2nd Edition, 2000.

[17] Holmes, P., Jenkins, J., Leonard, N.E., 'Dynamics of the Kirchhoff equations: Coincident centers of gravity and buoyancy'. Physica D 118, p. 311342, 1998.

[18] Biggs, J. D., Holderbaum, W., 'Planning Rigid body motions using Elastic curves'. Mathematics of Control, Signals, and Systems, Vol. 20, No. 4., pp. 351-367, Dec 2008.

[19] Biggs, J.D., Holderbaum, W. 'Optimal kinematic control of an autonomous underwater vehicle'. IEEE Transactions on Automatic Control, 54, (7), pp. 1623-1626, July 2009.

[20] Jurdjevic, V., 'Integrable Hamiltonian Systems on Complex Lie groups'. American Mathematical Society, Vol. 178, No. 838, 2005. 
[21] Biggs, J. D., Holderbaum, W., Jurdjevic, V., 'Singularities of Optimal Control Problems on some 6-D Lie groups'. IEEE Transactions on Automatic Control, pp. 1027-1038, June 2007.

[22] Izmailova, O. V., Kozlov, V. V, 'Analytical properties of solutions for EulerPoincare equations on solvable Lie algebras'. Moscow University Mechanics Bulletin, vol. 51, no. 3, pp. 12-16, 1996.

[23] Lawden, D., 'Elliptic functions and applications'. Springer-Verlag, New York, 1989. 